\title{
Editorial
}

\section{MiRNAs in cancer: Non-coding RNAs as appealing biomarkers for malignancy}

\author{
Pier Jr Morin \\ Department of Chemistry and Biochemistry, Université de Moncton, 18 Antonine-Maillet Avenue, Moncton, New \\ Brunswick, Canada E1A $3 E 9$ \\ Tel.: +1 506858 4355; Fax: +1 506858 4541; E-mail: pier.morin@umoncton.ca
}

It is estimated that up to $50 \%$ of all human proteincoding genes are regulated by miRNAs. This family of non-coding RNAs, with $\sim 1500$ members reported up-to-date, can impact a variety of cellular processes including cellular proliferation, differentiation and apoptosis. It is not surprising that deregulated expression of selected miRNAs can thus promote cancer initiation and progression. Several miRNAs with oncogenic properties, termed oncomirs, as well as miRNAs with tumor suppressive capabilities have been identified in different malignancies and this list is growing at a rapid pace. MiRNA-based therapies are attractive partly due to the fact that these molecules can target multiple genes in different signaling pathways simultaneously. In addition to their therapeutic potential, miRNAs are released into the circulation and measurement of such species in plasma and serum samples highlights the possibility of leveraging these molecules as potential biomarkers for early cancer detection, prognostic assessment and evaluation of therapeutic response in cancer patients.

In this issue of Cancer Biomarkers, Lodewijk et al. discuss differentially expressed miRNAs in subtypes of thyroid cancer: papillary, follicular, anaplastic and medullary thyroid carcinomas. A special interest is placed on the diagnostic potential of miRNAs in this malignancy. The current method utilized to characterize thyroid nodules consisting of fine needle aspiration biopsies is described. Unfortunately, $30 \%$ of such biopsies are inconclusive and a hemithyroidectomy is required. Their article thus delves into the potential usefulness of identifying a miRNA signature that would help avoid such a procedure and assist clinicians in discriminating between tumor and benign tissues as well as between tumor types.

Similarly, Hogan et al. describe in this issue a need for the identification of novel biomarkers in the early detection of colorectal cancer (CRC). 5-year patient survival is over $90 \%$ when detected at an early stage compared with less than $10 \%$ survival for stage IV patients. While advantages exist with the current methods employed for CRC detection, sensitivity and specificity challenges are discussed and the diagnostic potential of miRNAs is assessed. MiRNA stability and their presence in body fluids including feces make them attractive molecules for the development of noninvasive detection methods for this malignancy.

Much effort is also directed towards the identification of a circulating miRNA signature in glioblastoma multiforme (GBM), an aggressive type of brain tumor with dismal prognosis. In this issue, Odjélé et al. review the progress made in recent years on identifying miRNAs that would assist clinicians in diagnosing this malignancy. MiRNA levels of miR-128 and miR$342-3 p$ vary significantly in blood samples of GBM patients when compared with healthy subjects and are thus emerging as early favorites in the search for a GBM-associated miRNA signature. The article also looks at studies recently undertaken in GBM cell and rodent models that showed promising results on the use 
of miRNAs either as standalone therapeutic approach or as sensitizing agents when combined with hallmark chemotherapeutic drugs currently use to treat GBM patients.

Other review articles in this issue further highlight the diagnostic and therapeutic potential of miRNAs in different malignancies. Sand et al. describe the potential role of miRNAs in non-melanoma skin cancer (NM$\mathrm{SC}$ ); basal cell carcinoma and cutaneous squamous cell carcinoma, as well as identify relevant signaling pathways likely targeted by differentially expressed miRNAs. They offer a list of recommendations to improve NMSC-related miRNA research moving forward and several points from their list will appeal to researchers working with miRNAs in other malignancies. Wang et al. present miRNAs with diagnostic and prognostic utility in gastric cancer. Interestingly, they describe a subset of five miRNAs that can modulate multidrug resistance via Bcl-2 targeting further highlighting the potential relevance of these molecules to the processes of drug response and drug resistance assessment.
Harquail et al. wrap up our special issue by taking a closer look at miRNAs involved in epithelialmesenchymal (EMT) and mesenchymal-epithelial (MET) transitions. With a special focus on breast cancer, their review provides a detailed account of miRNAs underlying EMT/MET dynamics and further highlights the molecular basis associated with these processes in tumor metastasis.

Clearly, the discovery of miRNAs has lead to exciting opportunities for cancer therapy and diagnosis. Their regulation of key molecules and signaling pathways involved in cancer-related processes as well as their stability in body fluids further reinforce their attractiveness as therapeutic targets and biomarkers in cancer. While several hurdles, including efficient delivery and potential off-target effects, remain to be surpassed before miRNA-based therapies reach the clinic, recent advances in the field warrant a closer look at the potential roles that these molecules could play in cancer. 